\title{
Clarkson syndrome: a rare disease for approaching therapeutics in an unconventional way
}

National Research Council of Italy, Dipartimento di Elettronica, Informazione e Bioingegneria, Politecnico Piazza Leonardo da Vinci 32, 20133 Milano, Italy

\begin{abstract}
Diego Liberati*
Everybody is aware that big pharma could not afford to deal with every syndrome, especially if few tens of known subjects affected has been known since it was first described [1]. But so called Idiopathic Systemic Capillary Leak Syndrome (Clarkson's Disease) sometimes happens, even to somebody you happen to know. The only hope to find something useful within the few years usually left by the syndrome may be to try to make a collective thinking effort, for instance fast publishing everybody's thoughts in order to create an inexpensive (besides our own time) network hopefully yielding some advances. I will start to point out the little I know, hoping it will be a stimulus for somebody else to go further, and hopefully so on. I have to thank a dear friend, who has recently got affected by the syndrome and besides trying to fight it with the great help of the public health structure, being a great scientist himself is also willing to contribute to the understanding of the syndrome for the benefit not just of himself: chapeau!
\end{abstract}

Second, I have to thank professor Marco Cicardi at University of Milano: he happens to be one of the few in the whole world having devoted years of study to this very pathology, and he is involved in supervising the course of a few known cases here around. He provided me with a nice priming about the disease together with the reference to Xie et al. [2], apparently still the state of the art of what it is known about. One of his young co-workers, Maddalena $\mathrm{Wu}$, whom I am warmly indebted too, did patiently correct all the main errors I was making trying to hurry to a possible solution. Four high school studentsboth with classic (Anna Lunghi and Clara Tacconi) and scientific (Davide Mapelli and Alessandro Rizzo) majors-have spent many hours brainstorming with me about in fulfilling a new requirement in Italian high school-named school-work alternating-aiming to have them experimenting a few kind of possible future works while still enrolled in the standard curriculum: they have been so proactive and collaborative that the following few lines would probably not have got out of my thoughts.

In extreme synthesis:

- Causes are still unknown [1]

- Symptoms appear cyclically with acute phases [1]

- The capillary permeability seems to be due to vascular plates contrac6on [3]

- Common symptoms-due to abnormal vascular permeability [3]-are: -massive daily weight and related diuresis variations -generalized edema

- The non-apoptotic pathogen is present only in episodic serum that irritate also healthy cells [2]

- Most patients present a monoclonal immunoglobulin in plasma [2]
Interestingly enough, a possible gender disparity among light chains in monoclonal IgG is apparent even within the few known cases. In fact, there seems to be a different distribution of the kind of light chain $(\kappa$ or $\lambda)$ in immunoglobulins in males and females [2].

Variables easy to be measured by patients themselves during standard daily life are weight, diuresis, heart rate. Diuresis course appears to be very similar, just one day delayed, with respect to weight course, especially in the untreated phase in the considered patient, while their correlation with hearth rate seems to be less strong, probably because hearth rate changes could be less specific to the disease phases, besides being more fast varying.

If one thus concentrate on weight, the untreated patient seems to mainly differ from normal both for amplitude of daily oscillations (even few kilos instead of the physiological few hectograms) and for dominant period tending more to circa-septan than the usual almost circadian in normal.

When recording healthy females similar phenomena seems to appear: while half of the menstrual cycle is apparently closer to normal, the other half appears a bit more similar to the patient, even if with smaller amplitude and lower-but higher than daily-period of oscillation. In particular, a pair of females-single mother and daughter, having always lived with the mother, thus possibly synchronized-do present such bipartition synchronized in the first month of recording: of course it is necessary to have more longitudinal support together with much more subjects (but maybe there are data bases of daily weight for a number of normals, including females) to draw statistical inference, but such course is at least suggestive: could the disease be related to some (sexual) hormone-or its carrier in blood normally insulating it form vascular walls-disfunction yielding capillary leaking? This is a first possible hypothesis, it would be nice to have a follow up maybe on this same Journal in order to try to make a network yielding to a more concrete understanding, thus helping toward therapeutics and maybe tolerable drug also for such very rare disease. If it could work, that could be a complementary path to the traditional drug discovery process, thanks to the new ICT technologies also allowing open Journals!

\section{References}

1. Clarkson B, Thompson D, Horwith M, Luckey EH (1960) Cyclical edema and shock due to increased capillary permeability. Am J Med 29: 193-216. [Crossref]

Correspondence to: Diego Liberati, National Research Council of Italy, Dipartimento di Elettronica, Informazione e Bioingegneria, Politecnico Piazza Leonardo da Vinci 32, 20133 Milano, Italy, Tel: 393480569317; E-mail: diego. liberati@polimi.it

Received: September 28, 2017; Accepted: October 17, 2017; Published: October 20,2017 
2. Kapoor P, Greipp PT, Schaefer EW, Mandrekar SJ, Kamal AH, et al. (2010) Idiopathic Systemic Capillary Leak Syndrome (Clarkson's Disease): The Mayo Clinic Experience. Mayo Clin Proc 85: 905-912. [Crossref]
3. Xie Z, Ghosh CC, Patel R, Iwaki S, Gaskins D, et al. (2012) Vascular endothelial hyperpermeability induces the clinical symptoms of Clarkson disease (the systemic capillary leak syndrome). Blood 119: 4321-32. [Crossref]

Copyright: $\odot 2017$ Liberati D. This is an open-access article distributed under the terms of the Creative Commons Attribution License, which permits unrestricted use, distribution, and reproduction in any medium, provided the original author and source are credited. 